\title{
GEOMETRIC HODGE STRUCTURES WITH PRESCRIBED HODGE NUMBERS.
}

\author{
DONU ARAPURA
}

Let us call a pure, and necessarily effective polarizable, Hodge structure geometric if it is contained in the cohomology of a smooth complex projective variety. A natural question is which polarizable Hodge structures are geometric? Our goals in this note are twofold. We first of all show that there are no numerical restrictions on geometric Hodge structures. That is for any set of Hodge numbers (subject to the obvious constraints imposed by Hodge symmetry and effectivity), there exists a geometric Hodge structure with precisely these Hodge numbers. The argument is fairly easy, but we hope that there is some pedagogical value in writing it out. Our example is contained in the cohomology of a power $E^{N}$ of a CM elliptic curve. Since the Hodge conjecture is known for such varieties, we get a slightly stronger statement, that our example is the Hodge realization of a Grothendieck motive. The second part of this note is less elementary and more speculative. We try to address the question about where all the two dimensional irreducible geometric Hodge structures come from. The answer, assuming the Hodge conjecture, is that they come from either from elliptic curves or motives over $\overline{\mathbb{Q}}$. Examples of the latter type include the Hodge structures constructed in the first section, and Hodge structures associated to modular forms discussed in the second. Although we do not have a good conjecture about how to describe all the two dimensional $\overline{\mathbb{Q}}$-motivic Hodge structures, the ones arising from motives over $\mathbb{Q}$ should be exactly the modular examples.

Coming back to part one, we should mention that Schreieder $\underline{\mathrm{Scr}}$ has solved the related, but more difficult, problem of finding a smooth projective variety with a prescribed set of Hodge numbers in a given degree $k$ (under suitable hypotheses when $k$ is even). This does imply the main result here for odd $k$. Nevertheless, the construction is different and somewhat more involved. We thank Greg Pearlstein for first bringing Schreieder's work to our attention, and the referee for other useful remarks.

\section{MAIN THEOREM}

We will work exclusively with rational Hodge structures below. It is worth observing that since the category of polarizable Hodge structures is semisimple M], we have:

Proposition 1.1. A geometric Hodge structure of weight $k$ is a direct summand of $H^{k}(X, \mathbb{Q})$ for some smooth complex projective variety $X$.

Theorem 1.1. Given a set of nonnegative integers $g^{k, 0}, g^{k-1,1}, \ldots g^{0, k}$, satisfying $k \geq 0$ and $g^{p, q}=g^{q, p}$, there exists a geometric rational Hodge structure $H$ with $\operatorname{dim} H^{p, q}=g^{p, q}$.

Partially supported by the NSF and at the IAS by the Ralph E. and Doris M. Hansmann fund. 
Lemma 1.1. The Tate structure $\mathbb{Q}(-n)$ is geometric for $n \geq 0$. Let $V_{1}$ and $V_{2}$ be geometric Hodge structures of weight $n_{1}$ and $n_{2}$ respectively, then $V_{1} \otimes V_{2}$ is geometric. If $n_{1}=n_{2}$, then $V_{1} \oplus V_{2}$ is also geometric.

Proof. We have $\mathbb{Q}(-n)=H^{2 n}\left(\mathbb{P}^{n}, \mathbb{Q}\right)$. By assumption, there exists smooth projective varieties $X_{i}$ such that $V_{i}$ is a summand of $H^{n_{i}}\left(X_{i}\right)$. Then $V_{1} \otimes V_{2}$ and $V_{1} \oplus V_{2}$ are summands of $H^{k}\left(X_{1} \times X_{2}\right)$ where $k=n_{1} n_{2}$ and $k=n_{1}=n_{2}$ respectively.

Lemma 1.2. For any $n>0$, there exists a geometric Hodge structure $G$ with $\operatorname{dim} G^{n, 0}=\operatorname{dim} G^{0, n}=1$ and the remaining Hodge numbers equal to zero.

Proof. Although, it is not difficult to prove by hand, it is a bit cleaner if we make use of basic facts about Mumford-Tate groups, cf [L, appendix B] or [M]. In order to spell things out as explicitly as possible, we work with the specific elliptic curve $E=\mathbb{C} / \mathbb{Z} i \oplus \mathbb{Z}$. This has complex multiplication by $K=\mathbb{Q}(i)$. Let $V=H^{1}(E, \mathbb{Q})$. We choose a basis $v_{1}, v_{2}$ for $V$ dual to the basis $i, 1$ of the lattice $\mathbb{Z} i \oplus \mathbb{Z}$. Then $d z=i v_{1}+v_{2}$ and $d \bar{z}=-i v_{1}+v_{2}$ determines the Hodge decomposition on $V \otimes \mathbb{C}$. We define a homomorphism $h$ of the unit circle into $G L(V \otimes \mathbb{R})$ by rotations

$$
h(\theta)=\left(\begin{array}{cc}
\cos \theta & -\sin \theta \\
\sin \theta & \cos \theta
\end{array}\right)
$$

Then $h(\theta) d z=e^{i \theta} d z$, and $h(\theta) d \bar{z}=e^{-i \theta} d \bar{z}$. Let $S M T(V)$ denote the Weil restriction

$$
\operatorname{Res}_{K / \mathbb{Q}} \mathbb{G}_{m}=\left\{\left(\begin{array}{cc}
a & -b \\
b & a
\end{array}\right) \mid(a, b) \in \mathbb{Q}^{2},(a, b) \neq(0,0)\right\}
$$

This is clearly the smallest $\mathbb{Q}$-algebraic group whose real points contain $\operatorname{im} h$. In other words, $S M T(V)$ is the special Mumford-Tate group or Hodge group of $V$. The Mumford-Tate group $M T(V)$ is defined similarly, as the smallest $\mathbb{Q}$-algebraic group whose real points contain the image of Deligne's torus $\mathbb{S}(\mathbb{R})=\mathbb{C}^{*}$. It works out to the product of $S M T(V)$ and the group of nonzero scalar matrices. The significance of this group comes from the Tannakian interpretation: $M T(V)$ is the group whose category of representations is equivalent to the tensor category generated by $V$. Since $M T(V)$ was described as a matrix group, it comes with an obvious representation $\rho: M T(V) \rightarrow G L(V)$. For an integer $n$, let $V_{n}$ be the representation of this group given by composing $\rho$ with the $n$th power homomorphism $M T(V) \rightarrow M T(V)$. Now let us suppose that $n>0$. Then $V_{n}$ is irreducible, and therefore simple as a Hodge structure. The elements $d z, d \bar{z}$ still give a basis of $V_{n} \otimes \mathbb{C}$, but now the circle acts by $d z \mapsto e^{i n \theta} d z$ and $d \bar{z} \mapsto e^{-i n \theta} d z$. Thus these vectors span $V_{n}^{n, 0}$ and $V_{n}^{0, n}$. We have an embedding $V_{n} \subseteq S^{n} V \subset V^{\otimes n}$ given by identifying it with the span of $v_{1}^{n}$ and $v_{2}^{n}$. Therefore the previous lemma implies that $V_{n}$ is geometric. Thus $G=V_{n}$ is the desired Hodge structure.

Lemma 1.3. Given integers $p>q \geq 0$, there exists a geometric Hodge structure $H(p, q)$ with $\operatorname{dim} H(p, q)^{p, q}=\operatorname{dim} H(p, q)^{q, p}=1$ and the remaining Hodge numbers equal to zero.

Proof. Let $G$ be the Hodge structure constructed in the previous lemma with $n=$ $p-q$. Then $H(p, q)=G \otimes \mathbb{Q}(-q)$ will satisfy the above conditions,. 
Remark 1.2. We can replace $E$ by any $C M$ elliptic curve $E^{\prime}$, and the same construction works. We denote the corresponding Hodge structure by $H_{E^{\prime}}(p, q)$. However, the method will fail for non CM curves, because the Mumford-Tate group will no longer be abelian.

Proof of theorem. We get the desired Hodge structure by taking sums of the Hodge structures constructed in lemma 1.3. and the appropriate number of Tate structures when $k$ is even. More explicitly

$$
H=\bigoplus_{p>q} H(p, q)^{\oplus g^{p q}} \underbrace{\oplus \mathbb{Q}(-k / 2)^{\oplus g^{k / 2, k / 2}}}_{k \text { even }}
$$

Remark 1.3. As the referee points out, this can also proved by invoking [A] but we prefer to keep the argument elementary and self-contained.

The proof actually shows that $H \subset H^{k}\left(E^{N}, \mathbb{Q}\right)$ for some $N$. We can use this fact to get the stronger conclusion stated in the introduction; it says roughly that the inclusion is also defined algebro-geometrically. In order to make a precise statement, we recall that a motive, or more precisely an effective pure motive with respect to homological equivalence, consists of a smooth projective variety $X$ together with an algebraic cycle $p \in H^{*}(X \times X, \mathbb{Q})$ such that $p \circ p=p[\mathrm{~K}$. The composition $\circ$ is the usual one for correspondences [F2, chap 16]. It follows that $p$ is an idempotent of the ring of Hodge endomorphisms $\prod_{i} \operatorname{End}_{H S}\left(H^{i}(X)\right)$. Thus we get a mixed Hodge structure $p\left(H^{*}(X, \mathbb{Q})\right)$ which is by definition the Hodge realization of the motive $(X, p)$. We quickly run up against fundamental difficulties. For instance, it is unknown, whether $H^{k}(X)$ is the realization of a motive for arbitrary $X$ and $k$. For this, we would need to know that the Künneth components of the diagonal $\Delta \subset X \times X$ are algebraic, and this is one Grothendieck's standard conjectures G. Fortunately, it is not an issue in our example, because the Hodge conjecture holds for $E^{2 N}[\mathrm{~L}$, appendix B, §3] and this implies Grothendieck's conjecture. Thus $H^{k}\left(E^{N}\right)$ is the realization of a motive. Since $H$ is a summand of $H^{k}\left(E^{N}\right)$, it is given by the image of an idempotent in $\operatorname{End}_{H S}\left(H^{k}\left(E^{N}\right)\right)$. By the Hodge conjecture, this is algebraic. Thus to summarize:

Proposition 1.2. The geometric Hodge structure H, given in the theorem, is the Hodge realization of a motive.

\section{Two dimensional geometric Hodge structures}

Our goal now is to obtain an understanding of where two dimensional geometric Hodge structures come from. We may as well assume that the structures are irreducible because otherwise they are just sums of Tate structures. Given a subfield $K \subset \mathbb{C}$, by a motive over $K$ we will mean a pair $(X, p)$, as described earlier, such that both $X$ and the algebraic cycle representing $p$ are defined over $K$.

Theorem 2.1. Let $H$ be an irreducible two dimensional geometric Hodge structure. Then, assuming the Hodge conjecture, there are two nonexclusive possibilities.

(a) $H=H^{1}(E)(-m)$, where $E$ is an elliptic curve and $m \geq 0$, or

(b) $H$ comes from a motive over $\overline{\mathbb{Q}}$. 
Proof. We recall that the level of $H$ is the largest value of $|p-q|$ such that $H^{p q} \neq$ 0 . If the level is 1 then after Tate twisting, we can assume that $H$ is of type $(1,0),(0,1)$ and of course still 2 dimensional. So it must coincide with $H^{1}(E)$ where $E=H /\left(H^{10}+H_{\mathbb{Z}}\right)$ for some lattice $H_{\mathbb{Z}} \subset H_{\mathbb{Q}}$. If the level of $H$ is greater than 1 , we obtain (b) from the next proposition.

Proposition 2.1. Assuming the Hodge conjecture, any geometric Hodge structure with no consecutive nonzero Hodge numbers comes from a motive over $\overline{\mathbb{Q}}$. In particular, this conclusion applies to two dimensional geometric Hodge structures of level at least two.

Proof. Fix a period domain $D$ parametrizing polarized Hodge structures with fixed Hodge numbers $h^{m, 0}=*, h^{m-1,1}=0, h^{m-2,2}=*, \ldots$ CMP. Pick a point $t_{0} \in D$ corresponding to a Hodge structure $H$ which lies in $H^{m}(X, \mathbb{Q})$, where $X$ is a complex smooth projective variety. Since the category of polarizable Hodge structures is semisimple, $H$ is the image of an idempotent $p \in \operatorname{End}_{H S}\left(\bigoplus_{i} H^{i}(X, \mathbb{Q})\right)$. By the Hodge conjecture, this can be lifted to an algebraic cycle that we also denote by $p=\sum n_{i} p_{i}$ on $X \times X$. The pair $(X, p)$ is defined over a finitely generated extension of $K / \overline{\mathbb{Q}}$. We can regard $K$ as the function field of a variety $S / \overline{\mathbb{Q}}$. After shrinking $S$ if necessary, we can assume it is smooth and that $X$ is the generic fibre (based changed to $\mathbb{C}$ ) of a smooth projective family $f: \mathcal{X} \rightarrow S$. We can also assume, after further shrinking, that the relative cycles $\mathcal{P}_{i} \subset \mathcal{X} \times_{S} \mathcal{X}$ given by the scheme theoretic closure of the components $p_{i}$ are flat over $S$. Let $\mathcal{P}$ denote the relative correspondence $\sum n_{i} \mathcal{P}_{i}$. Then the rank of the image of the action of the fibre $\mathcal{P}_{s}$ on $H^{*}\left(\mathcal{X}_{s}(\mathbb{C})\right)$ is constant as $s$ varies. It follows that $\operatorname{im}\left[\mathcal{P}: R^{*} f_{*} \mathbb{Q} \rightarrow R^{*} f_{*} \mathbb{Q}\right]$ defines a variation of Hodge structure over $S(\mathbb{C})$. Let $\pi: \tilde{S}(\mathbb{C}) \rightarrow D$ be the associated period map on the universal cover. Now comes the key point. With our choice of Hodge numbers, Griffiths transversality forces $\pi$ to be constant. Therefore we can realize $t_{0}$ by the motive determined by any pair $\left(\mathcal{X}_{s}, \mathcal{P}_{s}\right)$ with $s \in S(\mathbb{C})$. In particular, we can choose $s \in S(\overline{\mathbb{Q}})$.

We describe a few examples of Hodge structures arising from motives over $\overline{\mathbb{Q}}$. Suppose that $E$ is a CM elliptic curve. Then as is well known [Si, chap II, §2], $E$ is defined over $\overline{\mathbb{Q}}$. Thus the examples $H_{E}(p, q)$ constructed earlier all arise from motives over $\overline{\mathbb{Q}}$. These examples have CM, and so are rather special. Recall that a Hodge structure $H$ has CM if $S M T(H)$ is abelian. It turns out that any irreducible two dimensional polarizable Hodge structure of even weight has CM. To see this, observe that the special Mumford-Tate group would be abelian since it would have to contain $\mathrm{SO}_{2}$ as a maximal compact. (Our thanks to the referee for this remark.) It is not difficult to conclude, as a consequence, that two dimensional irreducible Hodge structures of even weight are of the form $H_{E}(p, q)$, for some CM elliptic curve $E$.

In addition to the $H_{E}(p, q)$, there is one very natural source of examples: modular forms. Let us explain the basic set up. For simplicity, we work with the principal congruence group $\Gamma(n)=\operatorname{ker}\left[S L_{2}(\mathbb{Z}) \rightarrow S L_{2}(\mathbb{Z} / n)\right]$ with $n \geq 3$, but there are several other natural choices. Recall that a weight $k$ cusp form $f \in S_{k}(\Gamma(n))$ is given by a holomorphic function on the upper half plane $\mathbb{H}$ satisfying

$$
f\left(\frac{a z+b}{c z+d}\right)=(c z+d)^{k} f(z), \quad\left(\begin{array}{ll}
a & b \\
c & d
\end{array}\right) \in \Gamma(n)
$$


and such that the Fourier expansion $f=\sum a_{j} q^{j}, q=e^{2 \pi i z / n}$, has only positive terms [DS]. The moduli space $Y(n)=\mathbb{H} / \Gamma(n)$ of elliptic curves with a full level $n$ structure is a fine moduli space, so it comes with a universal family $\pi_{n}: \mathcal{E}(n) \rightarrow$ $Y(n)$. This can be completed to a minimal elliptic surface $\overline{\mathcal{E}}(n) \rightarrow X(n)$ over the nonsingular compactification $j: Y(n) \hookrightarrow X(n)$. By a theorem of Zucker [Z, the intersection cohomology of the symmetric power

$$
H=I H^{1}\left(X(n), S^{k-1} R^{1} \pi_{n *} \mathbb{Q}\right)=H^{1}\left(X(n), j_{*} S^{k-1} R^{1} \pi_{n *} \mathbb{Q}\right)
$$

carries a pure Hodge structure of weight $k$. This Hodge structure turns out to be isomorphic to one constructed by Shimura [Z, $\S 12]$. It has only $(k, 0)$ and $(0, k)$ parts, and the $(k, 0)$ part is isomorphic to the space $S_{k+1}(\Gamma(n))$ of cusp forms of weight $k+1$. We note that $H$ is geometric, since it can be shown to lie the cohomology of a desingularization of the $(k-1)$-fold fibre product $\overline{\mathcal{E}}(n) \times_{X(n)} \times \ldots \overline{\mathcal{E}}(n)$. The quickest way to see this is by applying the decomposition theorem for Hodge modules [Sa], although we won't actually need this. The space $X(n)$ has a large collection of commuting self-correspondences called Hecke operators DS. These operators act on $H[\mathrm{Sc}$, and they can be used to break it up into pieces. Suppose that $f=\sum a_{j} q^{j} \in S_{k+1}(\Gamma(n))$ is a suitably normalized nonzero simultaneous eigenvector for the Hecke operators, and further assume that the coefficients $a_{j}$ are all rational. Then $f$ and $\bar{f}$ span a two dimensional Hodge structure $H(f) \subset H$. These examples arise from motives over $\mathbb{Q}$ constructed by Scholl $\underline{\mathrm{Sc}}$. We want to argue that the converse is also true.

Given a smooth complex projective variety $X$, we can always choose a model $X_{k}$ over a finitely generated field $k$. Then in addition to singular cohomology $H^{*}(X, \mathbb{Q})$ with its Hodge structure, we can consider étale cohomology $H_{e t}^{*}\left(X_{\bar{k}}, \mathbb{Q}_{\ell}\right) \cong H^{*}(X, \mathbb{Q}) \otimes$ $\mathbb{Q}_{\ell}$ with its canonical $\operatorname{Gal}(\bar{k} / k)$-action [Mi]. Given a modular form $f$ of the above type, we can associate a Galois representation by replacing ordinary cohomology by étale cohomlogy in the discussion of the previous paragraph (c.f. D)). Serre $\mathrm{Se}$ conjectured, and Khare and Wintenberger $\mathrm{KW}$ proved, that a certain general class of two dimensional Galois representations agrees "modulo $\ell$ " with those coming from modular forms. Although we won't try to make this precise, we will spell out one key consequence [Se, thm 6]:

Theorem 2.2 (Serre, Khare-Wintenberger). Given a smooth projective variety defined over $\mathbb{Q}$ such that $H^{m}(X(\mathbb{C}))$ is two dimensional and of type $(m, 0),(0, m)$, then the Galois representation on $H_{e t}^{m}\left(X_{\overline{\mathbb{Q}}}, \mathbb{Q}_{\ell}\right)$ comes from a modular form of weight $m+1$.

If we assume Tate's conjectures $\mathrm{Ta}$, then this correspondence would have to come from an isomorphism of the underlying motives. In particular, it should be compatible with the Hodge structures. From here it seems a short step to conjecture the following:

Conjecture 2.3. Any two dimensional Hodge structure of odd weight associated to a motive over $\mathbb{Q}$ should be isomorphic to one given by a modular form.

This is hardly an original idea; a version of this is stated in the introduction to [FM], where it is referred to as a "well known conjecture". Having stated this conjecture, it seems a good idea to actually try to check it in some cases. A natural source of two dimensional Hodge structures, beyond those already considered, comes from the world of Calabi-Yau (or CY) threefolds. These are three dimensional smooth 
projective varieties $X$ with trivial canonical bundle and hence $h^{30}=1$. Usually one also requires $h^{10}=h^{20}=0$ but this plays no role here. We say that $X$ is rigid if it has no infinitesimal deformations, i.e. $H^{1}(X, T)=0$. By Serre duality, this is equivalent to the vanishing of $h^{12}=h^{21}$. Thus $H^{3}(X, \mathbb{C})=H^{30} \oplus H^{03}$ is two dimensional. There is by now a large collection of known examples of rigid CY's defined over $\mathbb{Q}$, see $\mathbb{M e}$. In this note we will be content to consider just one example. We recall that to a fan, by which we mean subdivision of a Euclidean space into rational polyhedral cones, we can associate a special kind of algebraic variety called a toric variety [F1]. This construction can be applied to a root lattice, where the Weyl chambers form a fan. Verrill $[\mathrm{V}$ ] constructed a rigid CY 3 -fold $V$ by first starting with the toric variety associated to the root lattice $A_{3}$, taking a double cover branched over an appropriate divisor in twice the anti-canonical system, and then choosing a suitable desingularization. It can be described more directly as a desingularization of the hypersurface

$$
(x+y+z+w)(y z w+z x w+x y w+x y z) t-(t+1)^{2} x y z w=0
$$

in $\mathbb{P}^{3} \times \mathbb{P}^{1}$ where $t$ an inhomogeneous coordinate on the second factor. Using this equation, Saito and Yui [SY, thm 5.1] gives an explicit birational equivalence between Verrill's variety $V$ and a desingularization $Y$ of the fibre product of the elliptic modular surface associated to the congruence group $\Gamma_{1}(6) \subset S L_{2}(\mathbb{Z})$ with itself. Since $h^{30}$ is a birational invariant, $h^{30}(Y)=1$. So under this birational equivalence $H^{3}(V)$ will correspond to the necessarily unique irreducible sub Hodge structure of $H^{3}(Y)$ of type $(3,0),(0,3)$. On the other hand $I H^{1}\left(X_{1}(6), S^{2} R^{1} \pi_{*} \mathbb{Q}\right) \subseteq H^{3}(Y)$ is also of this type. So these must match, and the conjecture is verified in this example.

\section{REFERENCES}

[A] S. Abdulali, Hodge structures of CM-type. J. Ramanujan Math. Soc. 20 (2005), no. 2, 155-162. [CMP] J. Carlson, S. Müller-Stach, C. Peters, Period mappings and period domains. Cambridge Studies in Advanced Mathematics, 85. Cambridge University Press, ( 2003)

[D] P. Deligne, Formes modulaires et representations $\ell$-adic, Sem. Bourbaki 355 (1968-69)

[DS] F. Diamond, J. Shurman, A first course in modular forms, Graduate Texts in Mathematics, 228. Springer-Verlag, New York, 2005.

[FM] J-M Fontaine, B. Mazur, Geometric Galois representations, Elliptic curves, modular forms, Fermat's last theorem (Hong Kong, 1993), 41 - 78, Ser. Number Theory, I, Int. Press, Cambridge, MA, 1995.

[F1] W. Fulton, Introduction to toric varieties. Annals of Mathematics Studies, 131. Princeton University Press, Princeton, NJ, 1993

[F2] W. Fulton, Intersection theory, Second edition. Ergebnisse der Mathematik und ihrer Grenzgebiete. Springer-Verlag, Berlin, 1998.

[G] A. Grothendieck, Standard conjectures on algebraic cycles, 1969 Algebraic Geometry (Internat. Colloq., Tata Inst. Fund. Res., Bombay, 1968) pp. 193-199 Oxford Univ. Press

[K] S. Kleiman, Motives, Algebraic geometry, Oslo 1970 (Proc. Fifth Nordic Summer-School in Math., Oslo, 1970), pp. 5382. Wolters-Noordhoff, 1972

[KW] C. Khare, J-P, Wintenberger, Serre's modularity conjecture, Proceedings of the International Congress of Mathematicians. Volume II, 280-293, Hindustan Book Agency, (2010)

[L] J. Lewis, A survey of the Hodge conjecture. Second edition. Appendix B by B. Brent Gordon. CRM Monograph Series, 10. American Mathematical Society, Providence, RI, 1999

[Me] C. Meyer, Modular Calabi-Yau threefolds . Fields Institute Monographs, 22. AMS (2005)

[Mi] J. Milne, Etale cohomology, Princeton University Press (1980)

[M] B. Moonen, Notes on Mumford-Tate groups, http://www.math.ru.nl/personal/bmoonen/

[SY] M-H. Saito, N. Yui, The modularity conjecture for rigid Calabi-Yau threefolds over Q. J. Math. Kyoto Univ. 41 (2001), no. 2, 403-419. 
[Sa] M. Saito, Modules de Hodge polarisables. Publ. Res. Inst. Math. Sci. 24 (1988), no. 6, 849 995 (1989).

[Sc] A. Scholl, Motives for modular forms, Invent. Math. 100 (1990), no. 2, 419 - 430

[Scr] S. Schreieder, On the construction problem for Hodge numbers, ArXiv 1301.0478v3.pdf

[Se] J-P. Serre, Sur les représentations modulaires de degré 2 de Gal $(\bar{Q} / Q)$. Duke Math. J. 54 (1987), no. 1, 179-230.

[Si] J. Silverman, Advanced topics in the arithmetic of elliptic curves. Graduate Texts in Mathematics, 151. Springer-Verlag, New York, 1994

[Ta] J. Tate, Algebraic cycles and poles of zeta functions. Arithmetical Algebraic Geometry (Proc. Conf. Purdue Univ., 1963) pp. 93-110 Harper \& Row, New York (1965)

[T] B. Totaro, Hodge structures of type $(n, 0, \ldots, 0, n)$, IMRN (2014)

[V] H Verrill, The L-series of certain rigid Calabi-Yau threefolds, J Numb. Theory, 81, (2000)

[Z] S. Zucker, Hodge theory with degenerating coefficients. $L^{2}$ cohomology in the Poincaré metric. Ann. of Math. (2) 109 (1979), no. 3, 415 - 476

Department of Mathematics, Purdue University, West Lafayette, IN 47907, U.S.A. 